\title{
Beyond Zoom: The New Reality
}

\author{
Brenda K. Wiederhold, PhD, MBA, BCB, BCN
}

A s 2020 DRAWs to a close, we find ourselves more than 9 months into weathering a global pandemic. In many ways, we have learned how to make the best of the situation. We have altered how we work, learn, and live. We have determined how to acquire necessities in the safest way possible, and we have even figured out how to have some fun along the way. However, the better part of a year into this crisis, we are also growing weary.

Many of us are beginning to find that the self-control and discipline that came so readily during the first months of the pandemic are becoming harder to muster. When under extreme stress, the human brain must work overtime to manage thoughts, feelings, and behavior. ${ }^{1}$ While it is beneficial to create short bursts of focused energy to extricate a person from a dangerous situation, it is difficult to maintain this level of heightened awareness for long periods of time. Pair this ongoing stress with the fact that many of us do not have access to our typical coping mechanisms (e.g., social gatherings, exercise classes, mental health care, etc.), and it is no wonder we are exhausted.

Faced with the prospect of many more months of the same, we need to look at what worked during the early days of shelter at home: namely, innovation. It is perhaps time to adapt again, to innovate in ways that can re-energize and rejuvenate our lives.

In the early days of the pandemic, when people were forced to shelter at home, they adopted any tool that would allow them to stay connected with their communities. For most, Zoom became a way of life. While only 10 million people attended Zoom meetings at the end of 2019 before coronavirus was widespread, by April 2020, usage had exploded to 300 million. $^{2}$ And for a while, this worked brilliantly. Zoom and other traditional videoconferencing software allowed people to connect and collaborate. It created the opportunity for a hint of normalcy in a suddenly and drastically altered landscape.

Yet, the ease and accessibility of arranging these calls meant that videoconference schedules quickly inflated, both within and beyond the workday, leading to burnout and the newly coined condition "Zoom fatigue." In fact, a recent study of a team of 350 Microsoft employees found that the average workweek jumped by four hours after the shift to remote work, and that employees were spending much of this time in meetings. ${ }^{3}$

At this pace, the novelty of videoconferencing soon wore off, and the significant issues with traditional videoconference programs became clear. Videoconferencing programs have not really changed much over the past 10-20 years, either in underlying technology or in user experience. ${ }^{4}$ The majority of the software that we currently use (games excluded) was not designed to mimic in-person social interaction closely. As a result, communication through these programs can feel quite "flat." While this was fine when we only used these programs occasionally, it has become a sizeable stumbling block now that we use them as a complete replacement for face-to-face interaction.

So, how can we create depth in a flat world? How can we bring life to traditional videoconferencing? The answer is virtual reality (VR).

Last week, I entered a Zoom meeting as an avatar that I created with an app called LoomieLive. I was able to upload a photo, personalize my avatar, and choose a unique background. I then went into a group meeting in Mozilla Hubs where we gathered as avatars for an hour-long discussion. Our new personas offered a welcome change from previous videoconferences.

Avatar-inhabited worlds aren't new. Twenty-five years ago, DigitalSpace produced some of the first Internet-based 3D avatar applications. I found them quite valuable to help treat patients with extreme shyness and social phobia. We were able to enter the virtual space together as avatars, allowing for anonymity as social skills, such as small talk and personal boundaries, were practiced. Then in 2003, SecondLife was launched. Through the subsequent decades, we've seen a growing list of these VR apps that mimic Zoom but with avatars and 3D virtual backgrounds, including Spatial, Mozilla Hubs, and Microsoft-owned AltspaceVR, each with their own bells and whistles. And why not? As this crisis has shown, traditional videoconferencing can only go so far toward re-creating in-person interactions. It is clear that continued refinement of these technologies may allow fully immersive VR during online meetings.

In contrast to the flat 2D world of most videoconferencing software, VR can provide an immersive experience-one with depth and a feeling of "being there," or presence. By capturing not only a person's facial expressions, but also their gestures and body language, VR creates a more visceral experience. People can wave or shake hands and even give a colleague a literal pat on the back. In addition, when users are able to move around within a virtual environment, rather than being confined to a Brady Bunch box on the screen, communication becomes more natural, more familiar, and even more collaborative. For example, design teams at Mattel use VR to review 3D designs of new toys, including 
Barbie's latest DreamHouse, and Pfizer uses it to orient and train new employees at manufacturing sites. ${ }^{5}$ These immersive elements of VR, added to more traditional videoconferencing, can help overcome some of the technical issues that cause Zoom fatigue, creating a more appealing, more effective means of communication.

Eventually, the pandemic will subside, and many employees will go back to the office either part- or full-time. This does not mean, however, that our reliance on technology will diminish. In fact, throughout the pandemic, our reliance on new technologies has only increased, and there is evidence that the trend will continue. A recent report predicted that the number of employees expected to wear augmented and mixed-reality headsets in the United States alone is projected to reach 8.6 million by 2028 - up from 25,000 in 2019. ${ }^{6}$ For now, we find ourselves on the precipice of a shift toward a mass adoption of VR and other advanced technologies. I look forward to seeing how VR continues to change how we work, how we play, and how we communicate.

\section{References}

1. Okon-Singer H, Hendler T, Pessoa L, et al. The neurobiology of emotion-cognition interactions: fundamental questions and strategies for future research. Frontiers in Human Neuroscience 2015; 9:58.
2. Wiederhold B. Connecting through technology during the Coronavirus disease 2019 pandemic: avoiding "Zoom fatigue." CyberPsychology, Behavior, \& Social Networking 2020; 23:437-438.

3. Microsoft Workplace. Microsoft analyzed data on its newly remote workforce. https://insights.office.com/workplaceanalytics/microsoft-analyzed-data-on-its-newly-remote-work force/ (accessed Nov. 4, 2020).

4. Ogrenich A. We launched a startup to combat Zoom fatigue. Remotely. https://medium.com/swlh/we-launched-a-startupto-combat-zoom-fatigue-remotely-3edc $94 \mathrm{fc} 4 \mathrm{e} 5 \mathrm{c}$ (accessed Nov. 4, 2020).

5. Stern J. You can go back to the office-you just need a virtual-reality headset. https://www.wsj.com/articles/you-cango-back-to-the-officeyou-just-need-a-virtual-reality-headset11601290813 ? mod=searchresults $\&$ page $=1 \&$ pos $=1$ (accessed Nov. 4, 2020).

6. Castellanos S. New hires to the holodeck: fidelity investments tries collaboration via virtual reality. https://www.wsj .com/articles/new-hires-to-the-holodeck-fidelity-investmentstries-collaboration-via-virtual-reality-11601890201 (accessed Nov. 4, 2020).
Brenda K. Wiederhold Editor-in-Chief 